\title{
Rapid Assessment of Tablet Film Coating Quality by Multispectral UV Imaging
}

\author{
Marten Klukkert, ${ }^{1}$ Jian X. Wu, ${ }^{2}$ Jukka Rantanen, ${ }^{2}$ Soenke Rehder, ${ }^{1}$ Jens M. Carstensen, ${ }^{3}$ \\ Thomas Rades, ${ }^{2}$ and Claudia S. Leopold ${ }^{1,4}$
}

Received 31 May 2015; accepted 13 September 2015; published online 13 October 2015

\begin{abstract}
Chemical imaging techniques are beneficial for control of tablet coating layer quality as they provide spectral and spatial information and allow characterization of various types of coating defects. The purpose of this study was to assess the applicability of multispectral UV imaging for assessment of the coating layer quality of tablets. UV images were used to detect, characterize, and localize coating layer defects such as chipped parts, inhomogeneities, and cracks, as well as to evaluate the coating surface texture. Acetylsalicylic acid tablets were prepared on a rotary tablet press and coated with a polyvinyl alcohol-polyethylene glycol graft copolymer using a pan coater. It was demonstrated that the coating intactness can be assessed accurately and fast by UV imaging. The different types of coating defects could be differentiated and localized based on multivariate image analysis and Soft Independent Modeling by Class Analogy applied to the UV images. Tablets with inhomogeneous texture of the coating could be identified and distinguished from those with a homogeneous surface texture. Consequently, UV imaging was shown to be well-suited for monitoring of the tablet coating layer quality. UV imaging is a promising technique for fast quality control of the tablet coating because of the high data acquisition speed and its nondestructive analytical nature.
\end{abstract}

KEY WORDS: multispectral UV imaging; multivariate image analysis; SIMCA; tablet coating quality; tablet quality attributes.

\section{INTRODUCTION}

Pharmaceutical tablets are commonly coated for reasons such as improvement of the visual appearance, facilitation of the tablet intake, taste masking, drug release control, and protection of the tablet core from light and moisture (1-5). Improper control of the coating quality can compromise active pharmaceutical ingredient (API) physical and chemical stability and may result in dose failure and dumping as well as an altered dissolution profile. Furthermore, inadequate appearance of the tablet coating reduces customer acceptance and patient compliance. In agreement with the standards specified by the regulatory authorities, film coating quality is routinely determined only with a small number of samples using, e.g., disintegration and dissolution tests as well as nonautomated

Electronic supplementary material The online version of this article (doi:10.1208/s12249-015-0414-x) contains supplementary material, which is available to authorized users.

${ }^{1}$ Division of Pharmaceutical Technology, Department of Chemistry, University of Hamburg, Bundesstraße 45, 20146, Hamburg, Germany.

${ }^{2}$ Department of Pharmacy, Faculty of Health and Medical Sciences, University of Copenhagen, Copenhagen, Denmark.

${ }^{3}$ Department of Applied Mathematics and Computer Science, Technical University of Denmark, Kongens Lyngby, Denmark.

${ }^{4}$ To whom correspondence should be addressed. (e-mail: claudia.leopold@uni-hamburg.de) inspection of tablet appearance in laboratories (6-8). Such quality control strategies are time-consuming and costintensive and follow the paradigm of measuring the quality from the product (7). Furthermore, these strategies do not allow to relate the obtained product characteristics to quality-relevant production parameters (7). Thus, inspired by the QbD and PAT initiatives of the regulatory authorities (911), pharmaceutical manufacturers are encouraged to build the quality into the product by understanding and mathematical relation of input variables to critical quality attributes (CQA) and control of the production process in real-time through the use of inline, fast, and nondestructive sensors. This strategy is beneficial because insufficient product quality is detected during the process, which can therefore be adjusted so that the product meets the defined specifications (9). In this context, vibrational spectroscopic techniques such as NIR (12-16) and Raman spectroscopy (17-19) have been demonstrated to be useful tools for evaluation of tablet coating quality as they are fast, nondestructive, and versatile and provide chemical as well as physical information on the samples. However, one drawback of such techniques is the lack of spatial information, as average spectra are obtained only from spots of a sample or from more than one tablet during a given integration time (20). Hence, for understanding and control of the film coating process, spatially resolved physicochemical information on the coated tablets can be beneficial $(21,22)$. The detection and localization of coating defects such as chipping at either the center or the edge of the tablet may 
be caused by different phenomena, such as twinning of tablets during film coating or insufficient flexibility of the coating film and thus allowing systematic adjustment of the defect-causing process parameters (4). In this context, spectral imaging techniques have been shown to be attractive for evaluation of tablet coating quality, as they are nondestructive and provide chemical as well as spatial information on the samples. For instance, coating integrity, thickness, and uniformity have been evaluated by hyperspectral NIR imaging (23-25) and terahertz pulsed imaging (TPI) (24,26-29). However, for real-time process monitoring, remaining challenges are the data acquisition speed (30) as well as the handling of the large data sets $(20,31)$. To obtain spectra with sufficiently high signal to noise ratio, many scans of each sampling point need to be recorded having a negative impact on the sampling time. In this context, the application of imaging techniques that allow capturing spectra of wavelength regions in which the sample exhibits a high absorptivity is attractive because it potentially enables acquisition of high-quality spectra within a short sensor integration time leading to an increased image acquisition speed (32). From the abovementioned perspective, the main goal of this study was to assess the suitability of six-wavelength UV imaging for evaluation of the coating quality of tablets. Multispectral UV imaging has been previously demonstrated to be a well-suited technique for evaluation of the API solidstate form within uncoated tablets (32). In the present study, it was investigated if defects such as chipped parts, cracks, and inhomogeneities in the coating layer can be detected, identified, and localized by UV imaging combined with multivariate image and pattern recognition analysis. Moreover, the possibility to determine the surface texture of the tablet coatings from the UV images was examined.

\section{MATERIALS AND METHODS}

\section{Materials}

Acetylsalicylic acid (ASA), highly dispersed silicon dioxide (Aerosil® 200), and potato starch all of $\mathrm{Ph}$. Eur. grade were obtained from Fagron (Barsbüttel, Germany). Microcrystalline cellulose (MCC, Avicel® PH-102) of Ph. Eur. grade was supplied by FMC Biopolymer (Dublin, Ireland), Kollicoat ${ }^{\circledR}$ IR (polyvinyl alcohol-polyethylene glycol graft copolymer (PVA/PEG)) of two colors (blue and yellow based on aluminum lakes) was donated by BASF (Ludwigshafen, Germany), and magnesium stearate (MgSt) by Baerlocher (Unterschleissheim, Germany).

\section{Methods}

\section{Tablet Compaction}

Tablet compaction was performed on a rotary tablet press (Fette 102i, Fette Compacting, Schwarzenbek, Germany) equipped with faceted punches of $8 \mathrm{~mm}$ diameter. ASA tablets $(50.0 \%(w / w)$ ASA, $40.5 \%(w / w)$ MCC, $8.0 \%(w / w)$ starch, $0.5 \%(w / w)$ silicon dioxide, and $1.0 \%(w / w) \mathrm{MgSt})$ with a weight of approximately $250 \mathrm{mg}$ were obtained at main compaction pressures of about $200 \mathrm{MPa}$ and a rotor speed of $20 \mathrm{rpm}$. All tableting experiments were performed in an airconditioned room at $21^{\circ} \mathrm{C}$ and a RH of $45 \%$.

\section{Pan Coating}

ASA tablets were film coated with a mixture of Kollicoat ${ }^{\circledR}$ IR (blue and yellow, resulting in a green color) using a pan coater (Solidlab 1, Bosch, Schopfheim, Germany) equipped with a nozzle of $1.2 \mathrm{~mm}$ diameter. During the coating process, the product temperature range was $32-46^{\circ} \mathrm{C}$, the air flow rate was varied between 53 and $61 \mathrm{~m}^{3} \mathrm{~h}^{-1}$, and the atomizing air pressure and fluid spray rate were set to $0.67 \mathrm{bar}$ and $1.0 \mathrm{~g} \mathrm{~min}^{-1}$, respectively. Under these intentionally nonideal process conditions, tablets with intact coating layer that show an either homogeneous or inhomogeneous surface texture as well as tablets with a defect coating layer (chipped parts or inhomogeneous appearance) were obtained. A small fraction of coated ASA tablets were manually processed with a scalpel to obtain tablets with cracks in the coating layer. Uncoated tablets were prepared by gently scraping of the coating layer.

\section{Photographic Imaging}

Photographs of all ASA tablets were taken with a 18megapixel Canon EOS 600D-SLR camera attached to a Canon EF-S 18-55 mm 1:3.5-5.6 IS II objective (both from Canon, Tokyo, Japan) and processed using Adobe ${ }^{\circledR}$ Photoshop ${ }^{\circledR}$ CS2 (ver. 9.0, Adobe Systems, San Jose, USA).

\section{Photometric Stereo}

Images of coated ASA tablets with either visually homogeneous or inhomogeneous coating were acquired using a Videometer MultiRay imager (Videometer, Hørsholm, Denmark) combined with the VideometerLab software (ver. 2.8, Videometer, Hørsholm, Denmark) to verify the surface texture analyzed by UV imaging. The instrument consists of a combined darkfield and coaxial brightfield illumination source which illuminates the sample at different angles. As wavelength $465 \mathrm{~nm}$ was selected, a silicon range imaging detector was used to collect the reflected light. The obtained images were of size $1280 \times 960$ pixels with a pixel size of $7.7 \mu \mathrm{m}$.

\section{UV Imaging}

UV imaging of all tablets was performed using a VideometerLabUV multispectral imager (Videometer, Hørsholm, Denmark) and the VideometerLab software (ver. 1.6, Videometer, Hørsholm, Denmark). The imager was equipped with a Mercury-Xenon UV light source and bandpass filters to illuminate the samples at six wavelengths $(254,280,300,313,334$, and $365 \mathrm{~nm})$ as well as a CCD camera to collect the light that is diffusely reflected by the samples. The field of view of the instrument with a size of $11.7 \mathrm{~cm} \times$ $8.8 \mathrm{~cm}$ was imaged within about $18 \mathrm{~s}$ and resulted in a raw data array of dimension $960 \times 1280 \times 6$, where the wavelengths are arranged along the third dimension. The spatial resolution was $77.9 \mu \mathrm{m}$.

The UV imager and the instrument setup are illustrated in Fig. 1. 

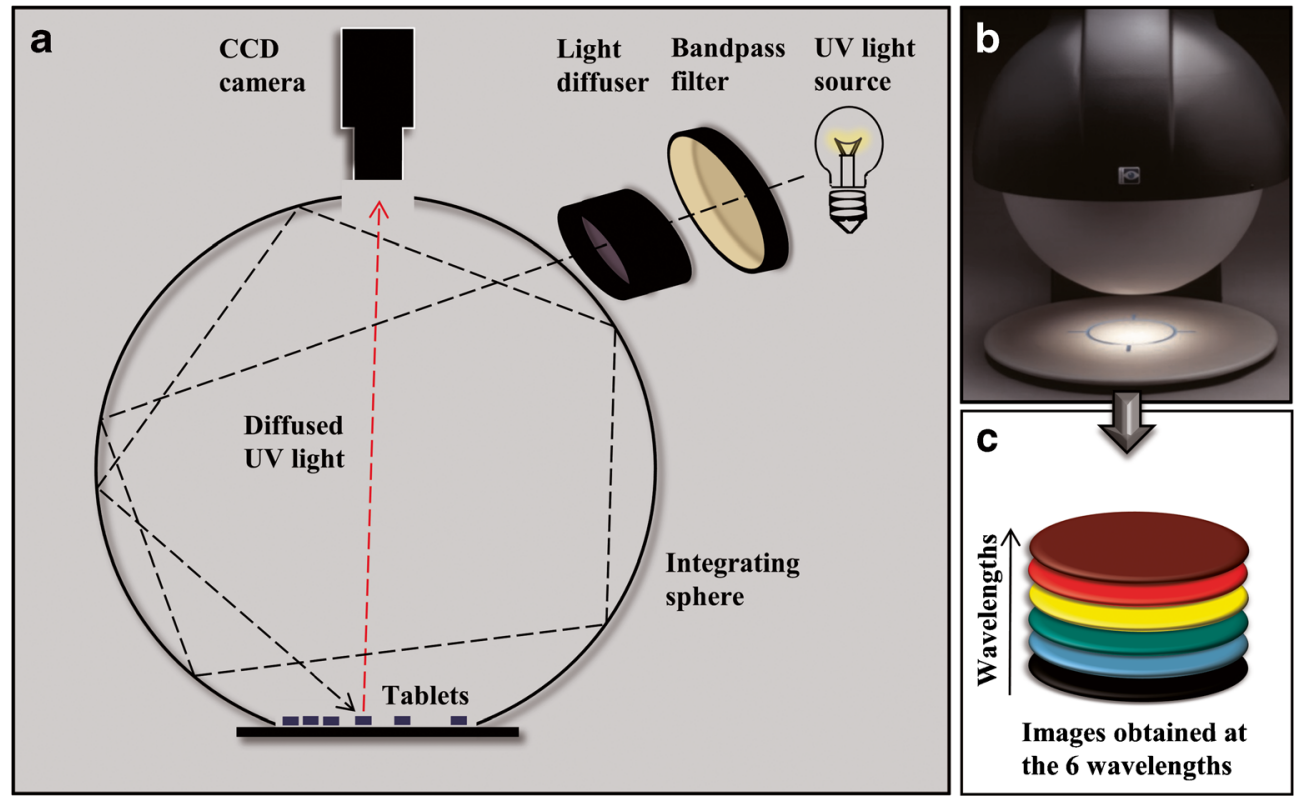

Fig. 1. a Schematic diagram of the UV imager. b Multispectral UV imager. c Data array obtained for each tablet

\section{Image Analysis}

Analysis of the UV images was based on principal component analysis (PCA), Soft Independent Modeling by Class Analogy (SIMCA) analysis, as well as related statistics and was performed with Matlab (ver. 8.1, Mathworks, Natick, USA) combined with the Image Processing Toolbox (ver. 8.0, Mathworks, Natick, USA) and the PLS_Toolbox (ver. 7.3, Eigenvector Research, Wenatchee, USA). In-house written Matlab scripts were applied to the images to evaluate intactness as well as the surface texture of coated ASA tablets.

To evaluate tablet coating layer intactness, ten reference tablets that are either uncoated or homogeneously coated as well as six test tablets that were uncoated or with a homogeneous, inhomogeneous, chipped, or cracked coating were analyzed. Prior to multivariate analysis, the UV images were transformed from reflectance $(R)$ to absorbance $(A)$ as follows (12):

$A=\log 10\left(\frac{1}{R}\right)$

Subsequently, tablet pixels were separated from the background by detection of the circular tablet structures in the 365nm UV images by Hough transformation (33). After erosion with a circle morphological structuring element with either three pixels (reference tablets) or one pixel (test tablets) in the radius, all remaining pixels within the circles were assigned to the respective tablet (34).

The UV spectra of all pixels of ten reference tablets which are either uncoated or homogeneously coated were used as an uncoated ASA tablet reference dataset and coating reference dataset, respectively. Global PCA (PCA model 1) was applied to both reference datasets to visualize the spectral variance within and between both groups of tablets (Table I).
To assign all pixels of the six test tablets to either the tablet coating or the uncoated ASA formulation based on their UV spectra, a SIMCA classification model was established based on the following two PCA models (Table I): one PCA model was applied to the mean-centered UV spectra of the uncoated ASA tablet reference dataset (PCA model 2) and another to the mean-centered UV spectra of the coating layer reference dataset (PCA model 3). Pixels were assigned to the class to which they were closest considering $\mathrm{T}^{2}$ and $\mathrm{Q}$ combined. The number of principal components (PCs) used in the PCA models (Table I) was chosen based on the root mean square error of cross validation (RMSECV) as a function of the PCs and minimized to obtain a robust classification model. The classification error of the SIMCA model was determined by calculation of the model sensitivity and specificity using the following equations (35):

Sensitivity $=\frac{\mathrm{tp}}{\mathrm{tp}+\mathrm{fn}}$

Specificity $=\frac{\mathrm{tn}}{\mathrm{tn}+\mathrm{fp}}$

where tp is the number of pixels which are known to be members of a respective class and that were correctly identified as class members, false negative (fn) is the number of pixels known to be class members that were incorrectly classified as nonmembers of the respective class, false positive (fp) is the number of pixels that do not belong to a respective class but are incorrectly classified as class members, and true negative ( $\mathrm{tn}$ ) is the number of pixels that are nonmembers of a respective class and that are correctly classified as being 
Table I. PCA Models Applied to the UV Images for Detection, Differentiation, and Localization of Coating Defects

\begin{tabular}{|c|c|c|c|}
\hline PCA model & 1 & 2 & 3 \\
\hline Dataset & $\begin{array}{l}\text { All pixels of five uncoated and } \\
\text { five intactly coated tablets }\end{array}$ & $\begin{array}{l}\text { Uncoated ASA tablets reference } \\
\text { dataset: all pixels of } \\
\text { five uncoated tablets }\end{array}$ & $\begin{array}{l}\text { Coating layer reference dataset: } \\
\text { all pixels of five intactly coated } \\
\text { tablets }\end{array}$ \\
\hline Purpose & $\begin{array}{l}\text { Visualization of the spectral } \\
\text { variance between uncoated and } \\
\text { intactly coated tablets as } \\
\text { well as between the respective } \\
\text { pixels of tablets with different } \\
\text { coating defects }\end{array}$ & $\begin{array}{l}\text { Model for PCA-based } \\
\text { SIMCA classification }\end{array}$ & $\begin{array}{l}\text { Model for PCA-based } \\
\text { SIMCA classification }\end{array}$ \\
\hline Related figure(s) & $\begin{array}{l}\text { Fig. } 2 b \text {, c } \\
\text { Fig. 3, column III and IV }\end{array}$ & Fig. 3, column V & Fig. 3, column V \\
\hline \multicolumn{4}{|l|}{ SIMCA } \\
\hline Used for SIMCA & No & Yes & Yes \\
\hline Model class & - & 1 & 2 \\
\hline $\begin{array}{l}\text { Number of PCs } \\
\text { included in the model }\end{array}$ & - & 2 & 1 \\
\hline Specificity & - & 1.0 & 1.0 \\
\hline Sensitivity & - & 1.0 & 1.0 \\
\hline
\end{tabular}

Prior to PCA, all spectral datasets were mean-centered

$P C A$ principal component analysis, $A S A$ acetylsalicylic acid, SIMCA Soft Independent Modeling by Class Analogy, PCs principal components

outside that class. Sensitivity is a parameter describing how accurately known class members are identified as such, while specificity is a parameter that indicates how well samples that are nonmembers of a respective class are classified as such (36). Specificity and sensitivity varies in the range between zero and unity. In general, values close to unity indicate a high classification performance of the model (37).

To distinguish tablets according to their coating texture by UV imaging, intact tablets that showed an either homogeneous $(n=8)$ or an inhomogeneous $(n=8)$ coating in the photographic images were analyzed as follows: in the first step, PCA-based background segmentation was applied (38). In the second step, pixels belonging to the facet of the tablets were assigned to the background by circular erosion of the tablet pixels using a circle morphological structuring element with 20 pixels in the radius (34).

From the segmented image, a global PCA was applied to all remaining pixels of the flat region of the 16 investigated tablets to monitor the spectral variance between them. For each tablet, the average score variation (Xa, Eq. 4) was calculated from the respective PC-1 scores as parametric estimator for coating surface texture by modification of the equation for calculation of the average roughness (39) as follows:

$\mathrm{Xa}=\sum_{n=1}^{N} \frac{\left|Z_{n}-\bar{Z}\right|}{N}$

where $Z_{n}$ is the individual PC-1 score intensity of each tablet pixel, $\bar{Z}$ is the mean PC-1 score intensity of all pixels of five coated tablets which were considered to show a homogeneous smooth coating (reference set), and $N$ is the total number of pixels of a respective tablet. The way of calculation of $\bar{Z}$ was based on the mean PC-1 score intensity of five homogenously coated reference tablets instead of that of the respective tablet, because tablets that are widely differing in surface texture could exhibit similar variation values if the score intensities spread comparably around the arithmetic average of the respective tablet (40). Thus, by calculating the average distance of the PC- 1 score intensities of the test tablets $\left(Z_{n}\right)$ from the mean score intensity of the reference set $(\bar{Z})$, solely those test tablets whose score intensities scatter around $\bar{Z}$ show a Xa value comparable to the homogenously coated reference tablets indicating a surface texture visually considered to be acceptable.

The mean Xa values of the five homogeneously coated reference tablets were calculated and set as $100 \%$. The $\mathrm{Xa}$ values of the remaining 11 tablets (test set) with a visually either homogeneous $(n=3)$ or inhomogeneous $(n=8)$ coating were calculated as percentage of the mean Xa value of the reference set. The data analysis procedures are explained in detail in the "Results and Discussion" section.

\section{RESULTS AND DISCUSSION}

\section{Detection, Differentiation, and Localization of Coating Defects}

To investigate the suitability of multispectral UV imaging to evaluate the integrity of the tablet coating layer, first of all it was investigated if uncoated and coated ASA tablets can be distinguished from each other based on their solid-state UV absorbance spectra. Therefore, mean UV spectra (Fig. 2a) of all pixels of an uncoated ASA tablet (Fig. 3, row A) as well as of a tablet with an intact coating and homogeneous smooth texture (Fig. 3, row B) were calculated from the respective UV images. It is obvious from Fig. 2a that the two types of tablets exhibit markedly different absorbance profiles that vary considerably with regard to the overall extent of UV absorbance as well as in the shape of the spectra. The tablet with the green-colored PVA/PEG coating layer (green curve) shows a markedly higher absorbance at all six wavelengths compared 


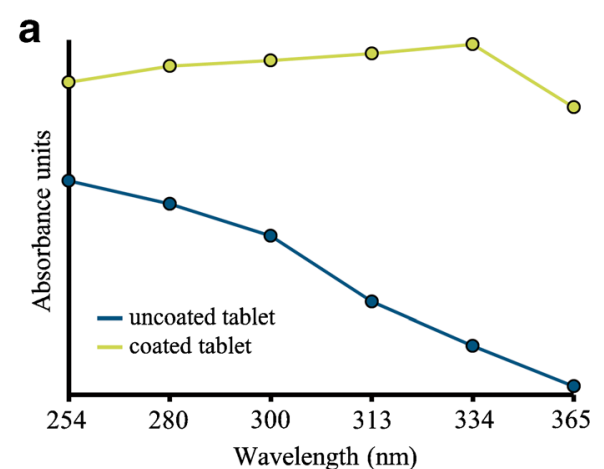

b
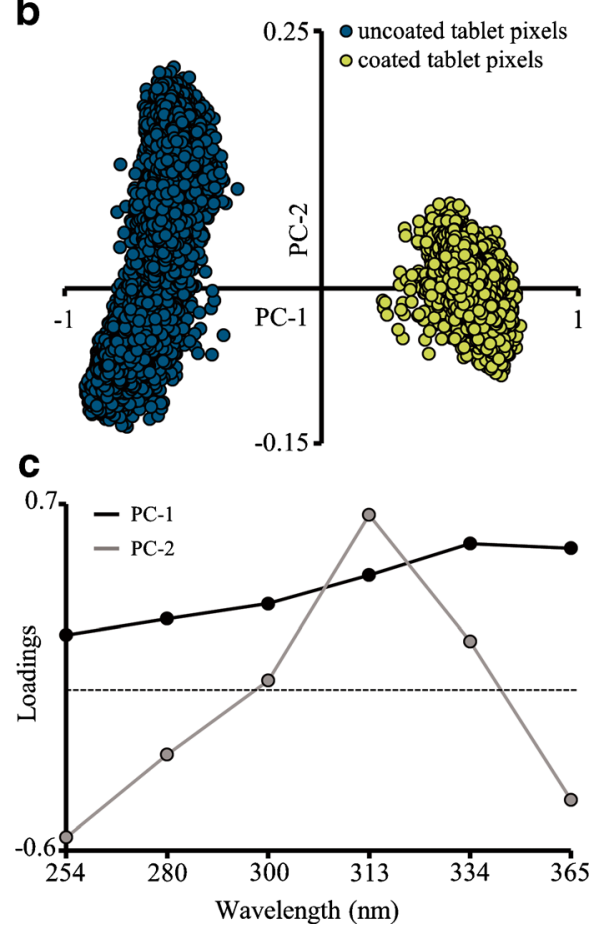

Fig. 2. a Mean UV absorbance spectra of an uncoated and a coated ASA tablet, respectively. b PCA (model 1) scatter plot applied to all pixels of uncoated and coated ASA tablets. c Loadings plot of PC-1 and PC-2

to the uncoated ASA tablet (blue curve) resulting in a baseline shift between the two spectra. Based on the chemical composition of the coating material, the coated ASA tablet shows a comparably featureless UV profile characteristic of the spectral response of the lakes in the coating formulation, while the uncoated tablet shows an increasing UV absorbance with decreasing wavelength due to the presence of ASA. To visualize the spectral variance of all pixels of a total of ten uncoated and intactly coated tablets (five tablets each), as well as to investigate if a systematic differentiation of the pixels of the two different tablet types is possible based on their UV spectra, PCA was applied to a reference dataset composed of all pixels of both tablet types (PCA model 1). PCA is a valuable technique for data reduction and visualization of the spectral features that contribute most to the variance within the spectral dataset $(41,42)$. Generally, samples that show similar spectra form clusters in the resulting score plot. As shown in Fig. 2b, all pixels of both the uncoated (blue dots) as well as the intactly coated ASA tablets (green dots) form clusters in the score plot which are clearly differentiated by PC- 1 that explains $98.6 \%$ of the spectral variance. All pixels of the coated tablets show a positive PC-1 score intensity, while pixels belonging to the uncoated ASA tablets form a cluster at negative PC-1 scores. In contrast, the pixels of both tablet types are not distinguished by PC-2 which captures only $1.1 \%$ of the spectral variance. Consequently, as shown in the corresponding loadings plot in Fig. 2c, PC-1 differentiates the pixels based on the observed differences in the shape of the pixel spectra of coated and uncoated ASA tablets, while PC-2 does not explain relevant spectral differences between uncoated and coated tablets. Thus, pixels belonging to the coated ASA tablets can be distinguished from those of uncoated tablets based on the UV spectra.

In a next step, the coating integrity of ASA tablets that are chipped at either the center or the edge of the tablet surface (Fig. 3, row C and D), of a tablet with an inhomogeneous coating (row E), as well as of a tablet with a crack in the coating layer (row F) was analyzed based on the respective UV images (Fig. 3, column II). Therefore, the spectral features of each pixel of the test tablets were extracted from the UV images and projected onto the PCA model which was previously built based on all pixels of the reference dataset composed of five uncoated and five intactly coated ASA tablets (PCA model 1). As shown in the resulting PCA score plot (Fig. 3, column III, row A), all pixels of the uncoated ASA test tablet are plotted next to the cluster or are even superimposed on the cluster related to the uncoated ASA reference tablets, which confirms complete absence of the coating layer. Furthermore, all pixels of the ASA tablet with an intact coating serving as test (Fig. 3, row B) form a cluster in direct vicinity of coating pixels (column III), which verifies intactness of the coating layer of the tablet. In contrast, the score plots of tablets with a partially chipped coating (column III, rows C and D) reveal that the majority of the tablet pixels are superimposed on the reference cluster of coating pixels, but there are also a number of nonclustered pixels with scores in between the two reference clusters and even pixels that are superimposed onto the uncoated ASA tablets reference cluster. These observations show that a considerable number of the pixels of the test tablet show spectral characteristics of both the coating layer as well as the uncoated ASA tablet or even spectra similar to the uncoated tablet. Thus, the PCA results correctly indicate that regions of the coating layer are chipped, which is accompanied by the appearance of the uncoated ASA tablet and thus leads to a decreased intensity of the PC-1 scores of the enclosing pixels. However, not all coating defects are accompanied by the appearance of the subjacent ASA tablet as shown by photographic images of tablets with an inhomogeneous coating caused by twinning of tablets during the coating process (Fig. 3, row E) or with a crack in the coating (Fig. 3, row F). The score plots resulting from subjecting the pixels of these tablets to the PCA model reveal that most of the pixels are superimposed on the coating pixel cluster with a small number of pixels showing lower PC-1 and higher PC-2 score intensities compared to the pixels of the intactly coated test tablet (Fig. 3, row B). It is hypothesized that these pixels surround the defect regions of the coating and show a slightly reduced absorbance compared to the smooth regions of the coat because of a partial discoloration of the coating layer in the regions of the defects. 


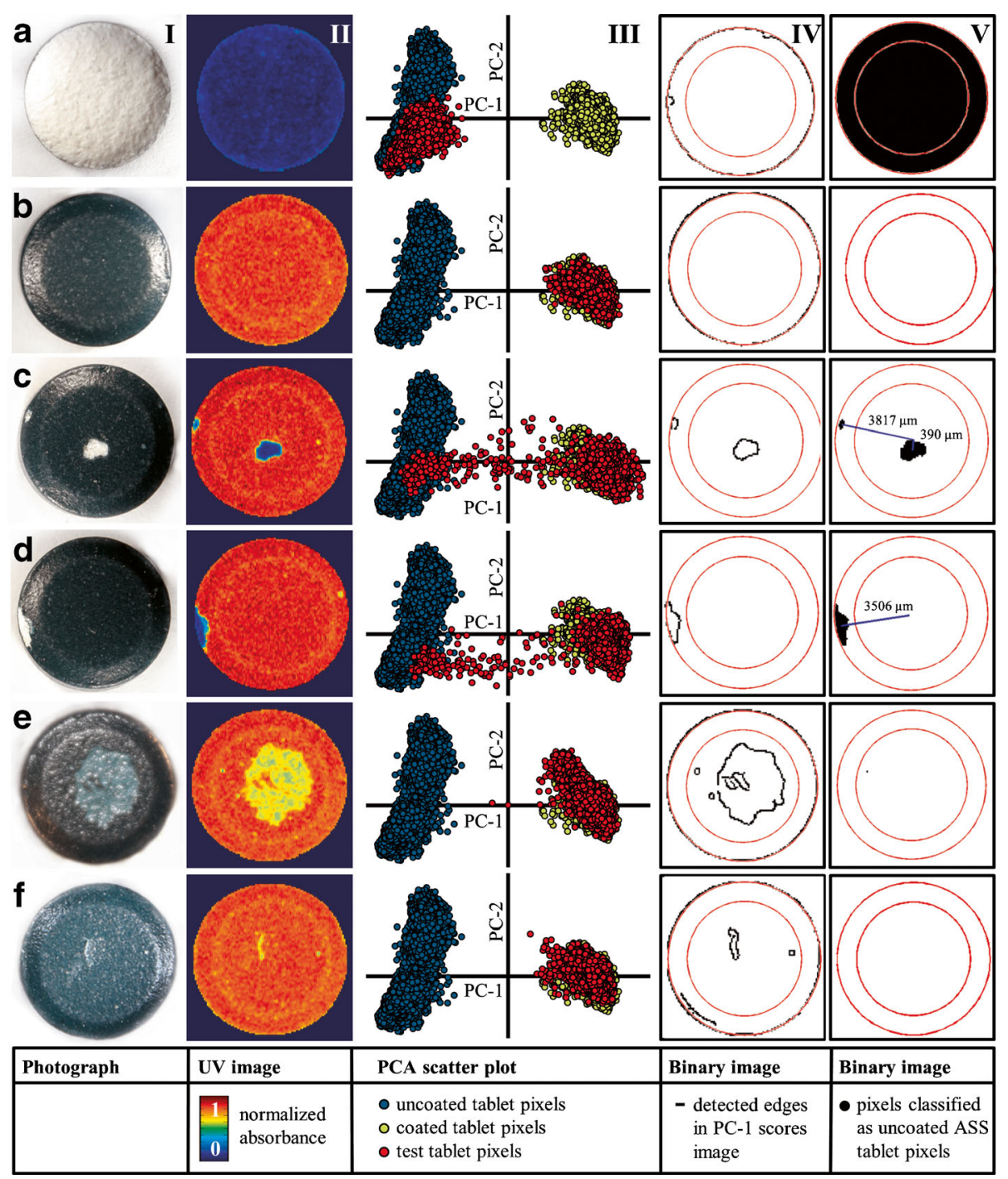

Fig. 3. Image analysis of representative ASA tablets with either homogeneous or inhomogeneous coatings. Columns from left to right correspond to photographic images (I), UV images at $365 \mathrm{~nm}$ (II), PCA (model 1) scatter plots (III), binary images obtained from application of an edge detection algorithm on PC-1 (PCA model 1) scores images (IV), and binary images with pixels assigned to the class of uncoated ASA tablet pixels (PCA model 2) being highlighted $(V)$

Consequently, pixels enclosing chipped coating regions are clearly separated from pixels of an intact coating by PCA, while pixels surrounding defects that are not accompanied by appearance of the subjacent ASA tablet can be hardly differentiated from pixels of an intact coating by analysis of the PCA score plots.

To achieve automatized detection, differentiation, and localization of the coating defects based on the UV images, a two-step image analysis routine was applied to the tablets shown in Fig. 3. First, to visualize all tablet coating defects, an edge detection algorithm (43) was applied to the score images (data not shown) of each tablet obtained from subjecting the test tablet pixels to the PCA model 1. In agreement with the UV images (Fig. 3, column II) as well as the previously obtained results from score plots of the tablets, pixels corresponding to the coating defects show a more or less altered UV spectrum compared to pixels enclosing an intact coating. Thus, the regions of such defects are being highlighted in the score images. The algorithm detects such structures caused by the difference in intensity of the PC- 1 scores between adjacent pixels at the edges of the defects. The resulting binary images with the detected edges being highlighted are shown in Fig. 3, column IV. It is obvious that the detected edges which neither correspond to the entire tablet nor to the tablet facet can either be assigned to minor inhomogeneities of the coatings (rows $\mathrm{E}$ and $\mathrm{F}$ ) or to the respective defects of the tablet coatings (rows C-F).

In a second step, to automatically distinguish between chipped coatings and coating defects that are not accompanied by appearance of the subjacent ASA tablet, a PCA-based SIMCA classification model was built with the purpose to assign each pixel of the test tablets that are shown in Fig. 3 to either the class of uncoated ASA tablet pixels (class 1) or the class of intact coating pixels (class 2). The SIMCA model was based on two PCA models (PCA model 2 and 3) calculated from the reference pixel datasets of either class 1 or 2 . Pixels that were assigned to the class of uncoated ASA tablet pixels (class 1) were automatically highlighted in binary 

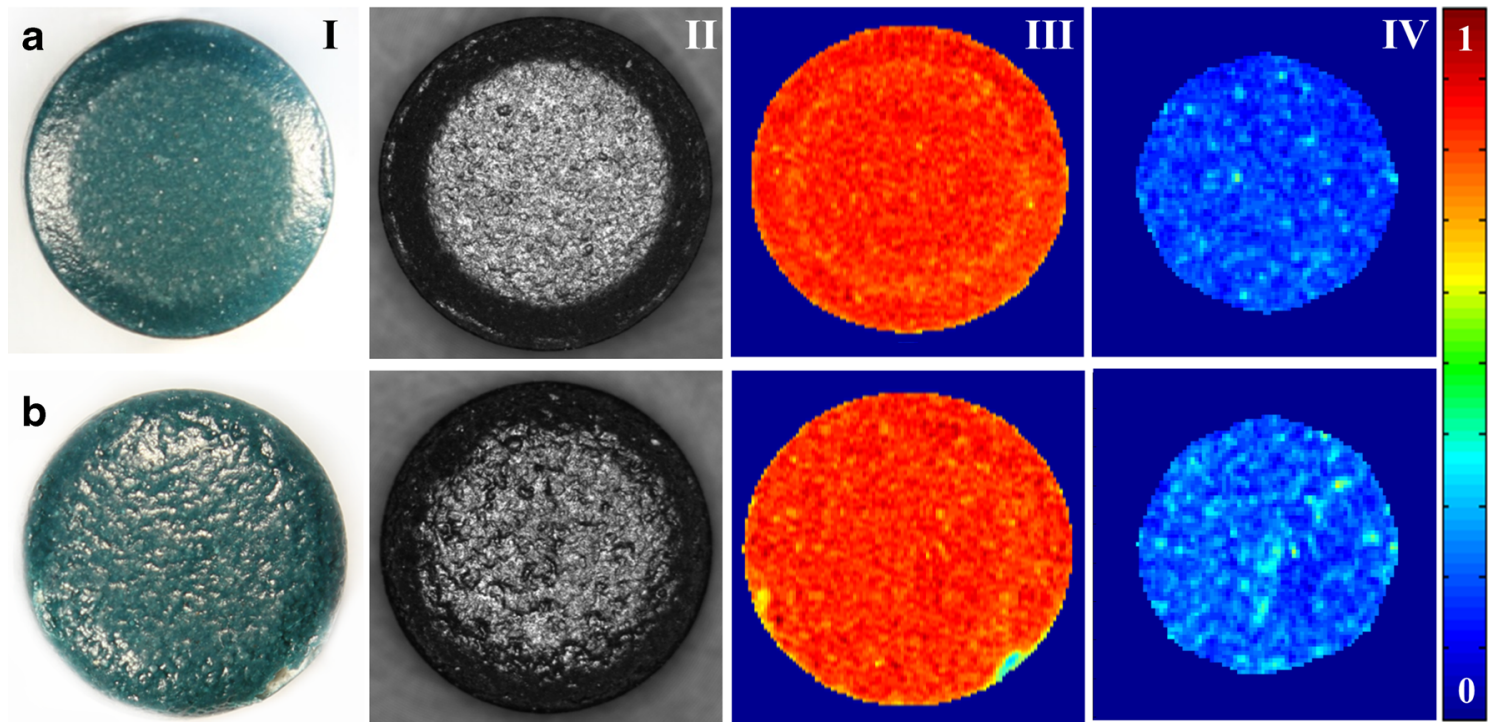

Fig. 4. Image analysis of representative ASA tablets with an acceptably homogeneous $(A)$ or an inhomogeneous $(B)$ coating texture. Columns from left to right correspond to photographic images (I), photometric stereo darkfield images (II), UV images at $365 \mathrm{~nm}(I I I)$, and PC-1 score images (IV)

images (Fig. 3, column V). It is obvious that, in agreement with the photographs and the UV images, no single pixel of the tablet with a uniform coating (row B) and of the tablet with cracked coating (row F) were assigned to class 1 . In contrast, all pixels of the uncoated ASA tablet (row A) as well as the pixels surrounding the pronounced coating defects at the center and edge of the tablets in rows $\mathrm{C}$ and $\mathrm{D}$ are correctly assigned to the class of uncoated ASA tablet pixels (class 1) and are thus highlighted in the binary images. As shown in the binary image of the tablet with an inhomogeneous coating (row E), all pixels except one, which could be removed by setting a threshold in the binary images based on the pixel quantity of highlighted pixel clusters, were correctly assigned to class 2 . Specificity and sensitivity (Eqs. 2 and 3) of the classification model which were calculated based on all pixels of the homogeneously coated and uncoated test tablets (rows A and B) are both 1.0 for both class 1 as well as class 2 indicating an adequate model quality. Thus, the defects of the investigated coated tablets that are accompanied by appearance of the subjacent ASA formulation could be clearly detected by a SIMCA classification model based on their multispectral UV images. Subsequently, localization of these defects was achieved by calculation of the distance from the center of the tablet to the center of the defects, which gives valuable information for readjustment of the coating process parameters. By comparing the defects found by application of either the edge detection algorithm or of the SIMCA model, a differentiation of coating defects that are not accompanied by appearance of the subjacent ASA tablet and defects resulting from chipped coatings parts could be achieved. It has to be mentioned that the detection of coating defects which are accompanied by the appearance of the subjacent tablet formulation are routinely performed by machine vision systems working in the VIS range. However, for a reliable detection of coating defects with VIS instruments, usually a high contrast in coloration between the coating layer and the tablet formulation is required. In contrast to VIS imaging, UV imaging is able to distinguish between different compounds based on their chemical nature irrespective of the contrast in coloration between them (32). As different chemical compounds show distinct UV profiles, this fact is considered to be a major benefit of UV imaging compared to systems working in the VIS range and it indicates the potential of UV imaging for the detection of defects even if the applied coating is of similar color (such as a moisture barrier coating) as the tablet formulation.

\section{Evaluation of Tablet Coating Texture}

A homogeneous surface texture accompanied by adequate appearance and gloss of the coating layer is usually a desired endpoint of the tablet coating process as the surface characteristics markedly affect product quality and performance (1). To investigate if UV images are suitable to distinguish between coated ASA tablets with the desired homogeneous surface texture (Fig. 4, row A) and those with an unacceptably inhomogeneous coating (Fig. 4, row B), representative coated tablets of both groups were chosen based on their visual appearance (Fig. 4, column I) and subsequently analyzed by UV imaging. It is well-known from the literature that spectral techniques working in diffuse reflectance mode are sensitive to variations in the surface texture of the samples $(44,45)$. Surface texture variations accompany spectral differences that manifest themselves in a spectral baseline offset rather than in a specific absorbance at a given wavelength $(41,46,47)$. To confirm the different surface textures of tablets that visually appear either homogeneous or inhomogeneous (Fig. 4, column I), in the first step darkfield images of the coated ASA tablets were captured by a photometric stereo imager to obtain a detailed surface map. It is obvious from the resulting images that the tablet visually considered to be smooth exhibits a rather homogeneous surface texture throughout the entire surface with small valleys and peaks on a microscale (Fig. 4, column II, row A). In contrast, the surface of the tablet considered to be unacceptably inhomogeneous clearly shows irregularities on a larger scale (Fig. 4, 
column II, row B), which is in agreement with the photographs. In Fig. 4, column III, the $365 \mathrm{~nm}$ channel UV images of the respective tablets are shown. To monitor the UV spectral variance throughout the coated ASA tablets in relation to each other in all six wavelengths of the UV images, PCA was applied to the UV image pixels of the central regions of 16 homogeneously $(n=8)$ and inhomogeneously $(z=8)$ coated tablets simultaneously. It is assumed that the variations in surface texture of the coated tablets cause major spectral differences that are consequently captured by the first PC and are visualized in the score images. As shown in Fig. 4, column IV, the PC-1 score intensity varies less throughout the surface of the tablet with a homogeneous coating (Fig. 4, row A) compared to the tablet with an inhomogeneous coating (Fig. 4, row B) and thus indicates a more even surface texture. As expected, the mean UV spectra of representative pixels with a low and high PC-1 score intensity mainly differ in the spectral baseline (Fig. 5a). Accordingly, the loadings plot of PC-1 (Fig. 5b) that explains $99.9 \%$ of the spectral variance
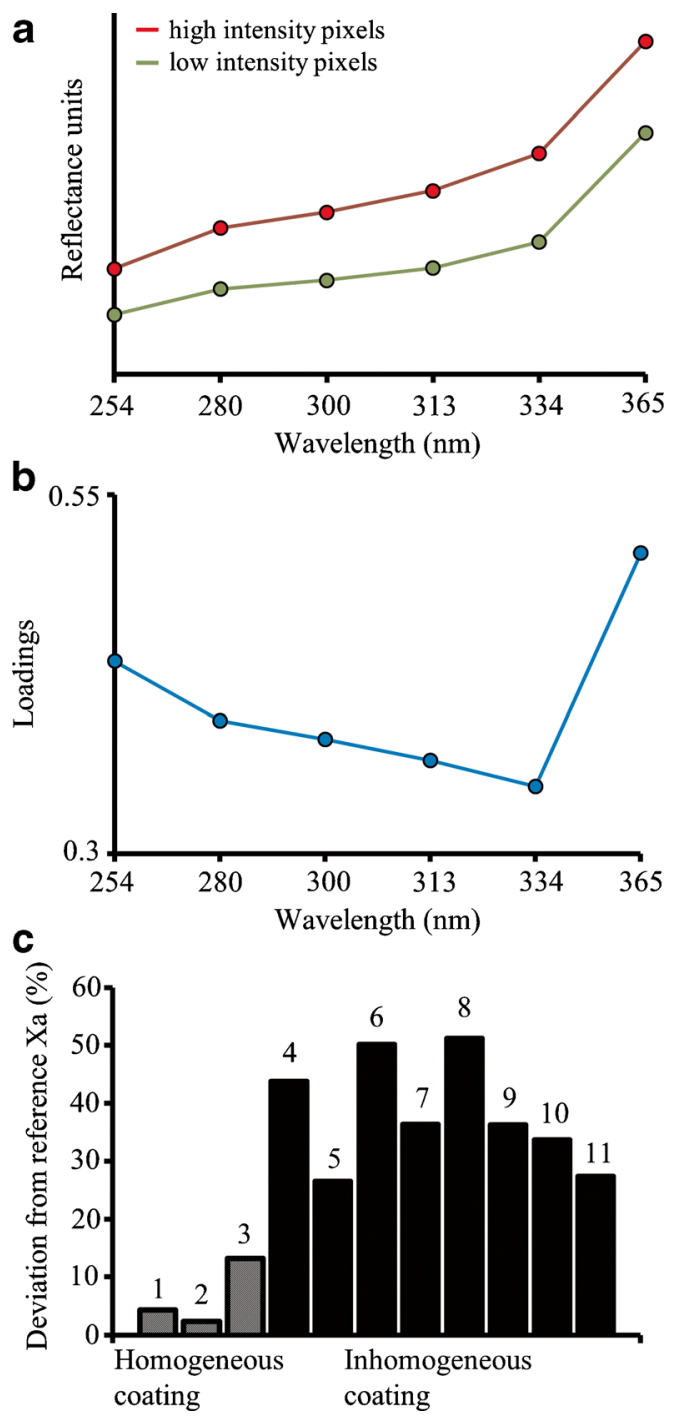

Fig. 5. a Mean UV spectra of high and low PC-1 score intensity pixels. b Loadings plot of PC-1. c Deviation of the average score variation (Xa) of 11 test tablets from the mean reference $\mathrm{Xa}$ of tablets with the desired coating texture mainly reflects the shape of the UV spectra and thus verifies that PC-1 captures spectral differences resulting from physical irregularities in the coating surface (48). To systematically compare the coating texture of the investigated tablets and to identify tablets that do not exhibit the desired homogeneous coating, the average score variation (Xa, Eq. 4) was calculated for each of 16 coated tablets from the respective PC-1 score images as estimate for the coating surface texture. A reference dataset composed of the PC-1 score images of five tablets with a homogeneous coating was used to calculate a mean Xa value which is representative for tablets with the desired coating profile. Subsequently, the PC-1 score images of another 11 tablets of the entire dataset with visually acceptable appearance (Fig. 5c, samples 1-3) and with an unacceptably inhomogeneous coating (Fig. 5c, samples 4-11) were analyzed by calculation of the deviation of the respective $\mathrm{Xa}$ from the mean $\mathrm{Xa}$ of the reference tablets $(=100 \%)$. It is obvious from Fig. 5c that tablets with a visually homogeneous coating show Xa values that are only about 3-13\% higher than the mean Xa value of the reference set (samples 1-3), while tablets with a visually inhomogeneous coating differ from the reference $\mathrm{Xa}$ by values between 27 and $51 \%$. Thus, tablets that exhibit an unacceptably inhomogeneous coating can be distinguished from those with a homogeneous coating by defining a limit of acceptable deviation from the mean $\mathrm{Xa}$ of tablets with desired coating texture. Thus, the estimation of the degree of coating surface homogeneity and thus the detection of insufficient coating quality was successfully based on six-wavelength UV images. It is worth to mention that because there is a systematic variation, i.e., a baseline shift, between the spectra in terms of texture analysis, a further reduction of the number of wavelengths, which would be accompanied by an increased sample throughput, might be possible to facilitate the measurement in the process environment. Such a reduction of the number of wavelengths can be justified if the analysis is started out with a higher number of wavelengths to establish a robust model and subsequently reduced in the number of wavelengths until the robustness and reliability of the model significantly decreases. However, such a reduction of the number of wavelengths has to be applied with caution, because the loading plot in Fig. 5 clearly suggests that all wavelengths are used to describe the underlying variation of the data (all wavelengths show a loading value being different from 0 ).

It is worth to mention that in this study analysis of the coating intactness and texture by UV imaging is based on different PCA models and texture analysis has only been applied to tablets that have previously shown to be intactly coated. Thus, the PCA model for texture analysis is trained particularly on intactly coated tablets and consequently provides reliable results only with regard to the coating texture of these tablets. To avoid misleading conclusions, it is essential that the two PCA models for evaluation of tablet coating intactness and for texture analysis are applied to the test tablets one after another.

\section{CONCLUSION}

Multispectral UV imaging allowed accurate and fast characterization of tablet coating layer intactness. Coating defects such as chipped parts, cracks, and inhomogeneities could be 
detected and analyzed by image analysis routines applied to the UV images of the coated ASA tablets. Tablets with intact coating and either homogeneous or inhomogeneous coating texture were successfully differentiated based on the UV images. The amount and relevance of the obtained data combined with a high-speed image acquisition makes UV imaging an attractive technique for at-line quality control of the coating process. In this context, the implementation of multispectral UV imaging in the manufacturing line of coated tablets has to be further investigated. Furthermore, although neither warming of the samples nor any visual changes of the tablet coating were observed after exposure of the samples to UV radiation during the measurements, the potential influence of UV radiation on curing of the tablet coating should be investigated in a further study.

\section{REFERENCES}

1. Rohera BD, Parikh NH. Influence of plasticizer type and coat level on surelease film properties. Pharm Dev Technol. 2002;7(4):407-20. doi:10.1081/Pdt-120015043.

2. Pearnchob N, Siepmann J, Bodmeier R. Pharmaceutical applications of shellac: moisture-protective and taste-masking coatings and extended-release matrix tablets. Drug Dev Ind Pharm. 2003;29(8):925-38. doi:10.1081/Ddc-120024188.

3. Siepmann F, Hoffmann A, Leclercq B, Carlin B, Siepmann J. How to adjust desired drug release patterns from ethylcellulosecoated dosage forms. J Control Release. 2007;119(2):182-9. doi:10.1016/J.Jconrel.2007.02.003.

4. Levina M, Cunningham CR. The effect of core design and formulation on the quality of film coated tablets. Pharm Technol Eur. 2005;17(4):29-37.

5. Sohi H, Sultana Y, Khar RK. Taste masking technologies in oral pharmaceuticals: recent developments and approaches. Drug Dev Ind Pharm. 2004;30(5):429-48. doi:10.1081/Ddc-120037477.

6. EDQM. European pharmacopoeia. 8th ed. Strasbourg: Council of Europe; 2013.

7. Yu LX. Pharmaceutical quality by design: product and process development, understanding, and control. Pharm Res. 2008;25(4):781-91. doi:10.1007/S11095-007-9511-1.

8. FDA. Code of federal regulations title 21. part 211. Current good manufacturing practice for finished pharmaceuticals. 2014. http:// www. accessdata.fda.gov/scripts/cdrh/cfdocs/cfcfr/ cfrsearch.cfm?cfrpart=211\&Showfr=1. Accessed 15 May 2015.

9. FDA. Guidance for industry pat-a framework for innovative pharmaceutical development, manufacturing, and quality assurance. 2004. http://www.fda.gov/downloads/drugs/guidances/ ucm070305. Accessed 15 May 2015.

10. FDA. Pharmaceutical CGMPs for the 21st century: a risk-based approach. 2004. http://www.fda.gov/downloads/drugs/ developmentapprovalprocess/manufacturing/ questionsandanswersoncurrentgoodmanufacturingpracticescgmpfordrugs/ucm176374. Accessed 15 May 2015.

11. ICH. Guideline on pharmaceutical development Q8 (2r). 2009. http://www.ich.org/fileadmin/public_web_site/ ich_products/guidelines/quality/q8_r1/step4/q8_r2_guideline. Accessed 15 May 2015.

12. Reich G. Near-infrared spectroscopy and imaging: basic principles and pharmaceutical applications. Adv Drug Deliv Rev. 2005;57(8):1109-43. doi:10.1016/J.Addr.2005.01.020.

13. Gendre C, Genty M, Boiret M, Julien M, Meunier L, Lecoq O, et al. Development of a process analytical technology (PAT) for in-line monitoring of film thickness and mass of coating materials during a pan coating operation. Eur J Pharm Sci. 2011;43(4):244 50. doi:10.1016/J.Ejps.2011.04.017.

14. Möltgen C, Herdling T, Reich G. A novel multivariate approach using science-based calibration for direct coating thickness determination in real-time nir process monitoring. Eur J Pharm Biopharm. 2013;85(3 Pt B):1056-63. doi:10.1016/ J.Ejpb.2013.09.011.
15. Kirsch JD, Drennen JK. Near-infrared spectroscopy: applications in the analysis of tablets and solid pharmaceutical dosage forms. Appl Spectrosc Rev. 1995;30(3):139-74. doi:10.1080/ 05704929508000906.

16. Andersson M, Folestad S, Gottfries J, Johansson MO, Josefson $\mathrm{M}$, Wahlund K. Quantitative analysis of film coating in a fluidized bed process by in-line nir spectrometry and multivariate batch calibration. Anal Chem. 2000;72(9):2099-108. doi:10.1021/ Ac990256r.

17. Müller J, Knop K, Thies J, Uerpmann C, Kleinebudde P. Feasibility of raman spectroscopy as pat tool in active coating. Drug Dev Ind Pharm. 2010;36(2):234-43. doi:10.3109/ 03639040903225109.

18. Romero-Torres S, Pérez-Ramos JD, Morris KR, Grant ER. Raman spectroscopic measurement of tablet-to-tablet coating variability. J Pharm Biomed Anal. 2005;38(2):270-4. doi:10.1016/ J.Jpba.2005.01.007.

19. Romero-Torres S, Pérez-Ramos JD, Morris KR, Grant ER. Raman spectroscopy for tablet coating thickness quantification and coating characterization in the presence of strong fluorescent interference. J Pharm Biomed Anal. 2006;41(3):811-9. doi:10.1016/J.Jpba.2006.01.033.

20. Gowen AA, O'donnell CP, Cullen PJ, Bell S. Recent applications of chemical imaging to pharmaceutical process monitoring and quality control. Eur J Pharm Biopharm. 2008;69(1):10-22. doi:10.1016/J.Ejpb.2007.10.013.

21. Zeitler JA, Taday PF, Newnham DA, Pepper M, Gordon KC, Rades T. Terahertz pulsed spectroscopy and imaging in the pharmaceutical setting-a review. J Pharm Pharmacol. 2007;59(2):20923. doi:10.1211/Jpp.59.2.0008.

22. Brock D, Zeitler JA, Funke A, Knop K, Kleinebudde P. Evaluation of critical process parameters for intra-tablet coating uniformity using terahertz pulsed imaging. Eur J Pharm Biopharm. 2013;85(3 Pt B):1122-9. doi:10.1016/ J.Ejpb.2013.07.004.

23. Cairós C, Amigo JM, Watt R, Coello J, Maspoch S. Implementation of enhanced correlation maps in near infrared chemical images: application in pharmaceutical research. Talanta. 2009;79(3):657-64. doi:10.1016/J.Talanta.2009.04.042.

24. Maurer L, Leuenberger H. Terahertz pulsed imaging and near infrared imaging to monitor the coating process of pharmaceutical tablets. Int J Pharm. 2009;370(1-2):8-16. doi:10.1016/ J.Ijpharm.2008.11.011.

25. Palou $\mathrm{A}$, Cruz J, Blanco $\mathrm{M}$, Tomàs $\mathrm{J}$, De Los Ríos J, Alcalà $\mathrm{M}$. Determination of drug, excipients and coating distribution in pharmaceutical tablets using Nir-Ci. J Pharm Anal. 2012;2(2):90-7. doi:10.1016/J.Jpha.2011.11.003.

26. Ho L, Müller R, Römer M, Gordon KC, Heinämäki J, Kleinebudde P. Analysis of sustained-release tablet film coats using terahertz pulsed imaging. J Control Release. 2007;119(3):253-61. doi:10.1016/J.Jconrel.2007.03.011.

27. Niwa M, Hiraishi Y, Terada K. Evaluation of coating properties of enteric-coated tablets using terahertz pulsed imaging. Pharm Res. 2014;31(8):2140-51. doi:10.1007/S11095-014-1314-6.

28. Fitzgerald AJ, Cole BE, Taday PF. Nondestructive analysis of tablet coating thicknesses using terahertz pulsed imaging. J Pharm Sci. 2005;94(1):177-83. doi:10.1002/Jps.20225.

29. Zhong S, Shen Y, Ho L, May RK, Zeitler JA, Evans M. Non-destructive quantification of pharmaceutical tablet coatings using terahertz pulsed imaging and optical coherence tomography. Opt Laser Eng. 2011;49(3):361-5. doi:10.1016/ J.Optlaseng.2010.11.003.

30. Amigo J. Emerging possibilities of near infrared spectroscopy and near infrared chemical imaging in the pharmaceutical manufacturing industry. The challenge of the process analytical technologies paradigm or just a research tool. NIR News. 2013;24(8):9.

31. Boldrini B, Kessler W, Rebner K, Kessler R. Hyperspectral imaging: a review of best practice, performance and pitfalls for inline and online applications. J Near Infrared Spectrosc. 2012;20(5):438.

32. Wu JX, Rehder S, Van Den Berg F, Amigo JM, Carstensen JM, Rades T, et al. Chemical imaging and solid state analysis at compact surfaces using UV imaging. Int J Pharm. 2014;477(12):527-35. doi:10.1016/J.Ijpharm.2014.10.064. 
33. Atherton TJ, Kerbyson DJ. Size invariant circle detection. Image Vision Comput. 1999;17(11):795-803. doi:10.1016/S02628856(98)00160-7.

34. Gonzalez RC, Woods RE, Eddins SL. Digital image processing using Matlab. 1st ed. Upper Saddle River: Prentice Hall; 2004.

35. Bylesjö M, Rantalainen M, Cloarec O, Nicholson JK, Holmes E, Trygg J. OPLS discriminant analysis: combining the strengths of PLS-DA and SIMCA classification. J Chemom. 2006;20(810):341-51. doi:10.1002/Cem.1006.

36. Derde MP, Massart DL. Comparison of the performance of the class modelling techniques UNEQ, SIMCA, and prima. Chemom Intell Lab Syst. 1988;4(1):65-93.

37. Forina M, Oliveri P, Casale M, Lanteri S. Multivariate range modeling, a new technique for multivariate class modeling: the uncertainty of the estimates of sensitivity and specificity. Anal Chim Acta. 2008;622(1-2):85-93. doi:10.1016/J.Aca.2008.05.065.

38. Vidal M, Amigo JM. Pre-processing of hyperspectral images. Essential steps before image analysis. Chemom Intell Lab Syst. 2012;117:138-48. doi:10.1016/J.Chemolab.2012.05.009.

39. Seitavuopio P, Heinämäki J, Rantanen J, Yliruusi J. Monitoring tablet surface roughness during the film coating process. Aaps Pharmscitech. 2006;7(2):E31. doi:10.1208/Pt070231.

40. Seitavuopio P. The roughness and imaging characterisation of different pharmaceutical surfaces. 2006. http://hdl.handle.net/ 10138/19122. Accessed 15 May 2015.

41. Wu J, Van Den Berg F, Rantanen J, Rades T, Yang M. Current advances and future trends in characterizing poorly water-soluble drugs using spectroscopic, imaging and data analytical techniques. Curr Pharm Des. 2014;20(3):436-53. doi:10.2174/ 13816128113199990398.

42. Lavine BK. Clustering and classification of analytical data. In: Meyers RA, editor. Encyclopedia of analytical chemistry. Chichester: JWiley; 2006 . doi:10.1002/ 9780470027318.A5204.Pub2.

43. Canny JA. Computational approach to edge detection. IEEE T Pattern Anal. 1986;PAMI-8(6):679-98. doi:10.1109/ Tpami.1986.4767851.

44. Ciurczak E. Uses of near-infrared spectroscopy in pharmaceutical analysis. Appl Spectrosc Rev. 1987;23(1-2):147-63. doi:10.1080/ 05704928708060445.

45. Gendrin C, Roggo Y, Collet C. Pharmaceutical applications of vibrational chemical imaging and chemometrics: a review: a review. J Pharm Biomed Anal. 2008;48(3):533-53. doi:10.1016/ J.Jpba.2008.08.014.

46. Ropero J, Colón Y, Johnson-Restrepo B, Romañach RJ. Nearinfrared chemical imaging slope as a new method to study tablet compaction and tablet relaxation. Appl Spectrosc. 2011;65(4):459-65. doi:10.1366/410-06078.

47. Rinnan A, Van Den Berg F, Engelsen SB. Review of the most common pre-processing techniques for near-infrared spectra. Trends Anal Chem. 2009;28(10):1201-22. doi:10.1016/ J.Trac.2009.07.007.

48. Eriksson L. Multi-and megavariate data analysis. 2nd ed. Umea: Umetrics AB; 2006 